\title{
Commentary
}

\section{Prevalence of tuberculosis, hepatitis C virus, and HIV in homeless people: a systematic review and meta-analysis}

\section{Antonio Cassone}

Invited Commentary on homelessness and infections

Homelessness is a widely universal and critical life condition that, whichever the underlying motivation (voluntary or forced), has profound health implications. Homeless people have little access to health services, including vaccination, and often suffer from underlying pathologies and mental disorders. Their morbidity and mortality are substantially higher than in the general population, and infections contribute to a substantial part of both morbidity and mortality. Of the estimated 100 million homeless people worldwide a remarkable proportion is represented by women and children. Poverty-related diseases such as those caused by Mycobacterium tuberculosis and HIV are associated with homelessness, and together with hepatitis $\mathrm{C}$, constitute a severe health risk for this population and, because of the contagion, for the general population as well. Sheltering may also be associated with increased risk of TB transmission. ${ }^{1}$ As for other minority populations such as illegal immigrants, refugees and displaced people, homeless population falls among the primary target of health interventions. However, for these interventions to be beneficial and cost-effective, an accurate estimate of disease prevalence is necessary.

In Lancet Infect.Dis., Beijer and collaborators ${ }^{2}$ have systematically reviewed published data on prevalence of tuberculosis, hepatitis C and HIV in homeless people. A meta-analysis was performed and appropriate statistics guided the assessment of difference significance in the published data. The expectation was that prevalence of TB could be higher in homeless people than in the general population of each selected country. What was less expected is the very high heterogeneity in prevalence of both TB and the other two analyzed diseases in different metaanalyzed studies, even in the same country (most of the studies were from the US). For instance, TB prevalence ratios ranged from 34 to 452 ( 0.2 to 7.7 percent prevalence). Interestingly, a meta-regression analysis suggested that different diagnostic methods such as chest radiography and sputum culture could be in part responsible for heterogeneity. Importantly, while the prevalence rate of TB reflected the prevalence ratios of the general populations, prevalence of HIV and hepatitis C did not, thus implying, as highlighted by the authors, that interventions related to provision of health services specifically to control these two illnesses in the general population may not be appropriate for disease control in homeless subjects. Of note, the meta-analysis essentially refers to data from rich countries, particularly USA and European countries. Overall, large prevalence heterogeneity suggests that health interventions in those countries need to be selective and adapted to each particular situation. For instance, intervention against TB in sheltered homeless ${ }^{3}$ may privilege a mandatory screening, and supervised housing appears effective in increasing treatment compliance resulting in substantial cost savings, a goal that can be much more difficult to reach and cost-ineffective for the unsheltered homeless.

The authors underscore the need for further studies that assess local factors distinguishing between the different aspects and conditions associated to homelessness, in particular transitory versus long-term homelessness. In this respect, data from poor countries where homelessness and displacement are more a rule than the exception would be a major contribution. Focusing health intervention, and human and financial resources on the specific target situation seems to be mandatory to reach efficacy and cost-effectiveness. This is an agreeable and wise conclusion. More naively, rich countries should make better efforts to provide easier access to non-marginal housing. Unfortunately, the first thing to do is often the hardest one.

\section{References}

1 Dobbins, C. et al. Tuberculosis Outbreak Associated with a Homeless Shelter - Kane County, Illinois, 2007-2011. MMWR. March 23, 2012/61(11);186-189.

2 Beijer, U., Wolf A, Fazel, S. Prevalence of tuberculosis, hepatitis $\mathrm{C}$ virus and HIV in homeless people : a systematic review and metaanalysis. Lancet Infect.Dis,........

3 Figueroa-Munoz J and Ramon-Pardo, P. Tuberculosis control in vulnerable groups. WHO Bulletin. 2008;86:9. 